Article

\title{
Nanoparticles and Single Atoms in Commercial Carbon-Supported Platinum-Group Metal Catalysts
}

\author{
Urša Petek $^{1,2}$, Francisco Ruiz-Zepeda ${ }^{1}$ (D), Marjan Bele ${ }^{1}$ and Miran Gaberšček ${ }^{1,2, *(D)}$ \\ 1 Department of Materials Chemistry, National Institute of Chemistry, Hajdrihova 19, \\ SI-1000 Ljubljana, Slovenia; ursa.petek5@gmail.com (U.P.); francisco.ruizzepeda@ki.si (F.R.-Z.); \\ marjan.bele@ki.si (M.B.) \\ 2 Faculty of Chemistry and Chemical Technology, University of Ljubljana, Večna pot 113, \\ SI-1000 Ljubljana, Slovenia \\ * $\quad$ Correspondence: miran.gaberscek@ki.si; Tel.: +386-1-4760-32
}

Received: 19 November 2018; Accepted: 30 January 2019; Published: 1 February 2019

\begin{abstract}
Nanoparticles of platinum-group metals (PGM) on carbon supports are widely used as catalysts for a number of chemical and electrochemical conversions on laboratory and industrial scale. The newly emerging field of single-atom catalysis focuses on the ultimate level of metal dispersion, i.e. atomically dispersed metal species anchored on the substrate surface. However, the presence of single atoms in traditional nanoparticle-based catalysts remains largely overlooked. In this work, we use aberration-corrected scanning transmission electron microscope to investigate four commercially available nanoparticle-based PGM/C catalysts (PGM = Ru, Rh, Pd, Pt). Annular dark-field (ADF) images at high magnifications reveal that in addition to nanoparticles, single atoms are also present on the surface of carbon substrates. Scanning electron microscopy, X-ray diffraction and size distribution analysis show that the materials vary in nanoparticle size and type of carbon support. These observations raise questions about the possible ubiquitous presence of single atoms in conventional nanoparticle PGM/C catalysts and the role they may play in their synthesis, activity, and stability. We critically discuss the observations with regard to the quickly developing field of single atom catalysis.
\end{abstract}

Keywords: single-atom catalysis; carbon-supported catalysts; platinum-group metals; aberration-corrected scanning transmission electron microscopy

\section{Introduction}

Catalysts that consist of metal nanoparticles dispersed on a supporting carbon material $(\mathrm{M} / \mathrm{C})$ are very commonly used in various fields of catalysis and electrocatalysis. Materials containing dispersed platinum-group metals (PGM) such as $\mathrm{Ru}, \mathrm{Rh}, \mathrm{Pd}$ and Pt are often used as heterogeneous catalysts in many reactions for chemical production [1,2]. $\mathrm{Pt} / \mathrm{C}$ is also an important electrocatalyst for oxygen reduction and hydrogen oxidation reaction in low-temperature fuel cells [3]. Due to their widespread use, there are many commercial PGM/C catalysts available on laboratory and industrial scales.

In the past decade, a new research field of heterogeneous catalysis emerged, namely, single-atom catalysis (SAC). Although atomically dispersed species of non-noble metals such as Fe, Co, and Ni on carbon supports were long studied as an alternative to Pt-based materials in fuel cells [4,5], the idea of single atoms of noble metals as counterparts to traditional nanoparticle-based catalysis is relatively recent [6-8]. This line of investigation became strongly promoted with the increasing availability of sub-ångström resolution aberration-corrected transmission electron microscopy (AC-STEM) [9,10] which made it possible to visualize single atoms dispersed on support. In terms of visual detection, carbon materials have an advantage over some other support materials (such as oxides) because of 
the large difference in atomic numbers $(Z)$ between the support and the dispersed transition metal. That enables an easier visualization of the dispersed metal with Z-contrast images compared to the supports that themselves contain atoms of other metals with similar $Z$ numbers.

SAC was initially regarded as a new frontier of nanoparticle-based heterogeneous catalysis. The properties and expected catalytic behaviours of single atoms were predicted by simply extrapolating size-dependent trends observed in nanoparticles to the ultimate limit of size reduction, i.e. atomic dispersion [6,7]. It was suggested that by achieving atomic dispersion, the efficiency of the metal use could be maximized, since all the metal atoms would be on the surface of the support and thus accessible to the reactants. At the same time, single atoms were expected to maintain or increase their activity as compared to active sites on the metal. Thus, SAC promised to offer a significant improvement in cost efficiency over traditional supported PGM catalysts where part of the atoms within the nanoparticles are not exposed to the surface and thus cannot serve as active sites. This strong motivation, coupled with increasingly accessible techniques for detection and visualization, helped single-atom catalysis become an established and growing research field within heterogeneous catalysis and electrocatalysis. The surge of interest in atomically dispersed metals significantly diversified the field by investigating many different transition metals, supporting materials and catalysed reactions [8,11-14].

Indeed, many atomic species on carbon and other hosts were synthesized and shown to possess high catalytic activity for various reactions $[15,16]$. However, with the growing number of experimental and theoretical results, those initial assumptions regarding the role of SAC are being investigated in more detail and, occasionally, partly reinterpreted. Publications reporting atomic dispersion on carbon supports show ionic nature of the single atoms and direct attachment of metal to atoms that are part of the carbon framework [15-17]. This shows that single atoms are fundamentally different chemical species than metallic atoms on nanoparticle surface. Trends observed for nanoparticle size-dependent behaviour cannot be expected to be generally valid when the particle size is reduced from nanoparticles to clusters and, finally, single atoms. Of course, this is also true for catalytic activity. Even though it was shown that in some cases, single atoms can catalyse the same reaction as nanoparticles, this behaviour cannot be expected as a general rule. Instead, it was recently suggested that atomically dispersed metals may have more similarities with analogous homogeneous catalysts than with supported nanoparticles [12]. Of course, the properties of a given supported metal atom are decisively defined by the exact nature of the single atom specie(s) present in the catalytic material (oxidation state, type of bonding with the support, bonding symmetry) and the nature of the support. Yet, as a rough generalization, a comparison of single atoms to homogeneous complexes seems more rational than a comparison with metallic surfaces.

Both the catalytic activity and the issue of stability of supported atomic species are sometimes compared to nanoparticles. It is well-understood that a high specific surface area of metal nanoparticles brings about a high surface energy, which is thermodynamically unfavourable $[18,19]$. Intuitively, it may be expected that single atoms should have a very high tendency to aggregate. However, this assumption ignores the chemical interactions of metallic atoms (ions) with anchoring sites on the support $[7,14,20]$. It should not be overlooked that the concept of "metallic surface" that dominates the properties of nanoparticles does not exist at the limit of atomic distribution. Instead, the surface properties of the carbon support before and after metal decoration may provide more relevant thermodynamic considerations.

Understanding similarities and, more importantly, differences between supported single atoms and nanoparticles and their behaviour toward the same reaction is especially important when both species coexist in the same catalyst material. Any observed catalytic behaviour (activity, selectivity) of a given catalyst is the overall sum of contributions of all the active species that occur in the material, including particles of the dispersed active phase of varying sizes and shapes, single atoms, the surface of the supporting material and possible impurities. In such complex systems, it is very difficult to identify the species responsible for the observed behaviour, especially since the properties of the 
catalysts tend to change along the duration of the observed catalytic process (stability). For this reason, in order to better understand the chemical nature of atomically dispersed species and their catalytic behaviour, there has been a lot of effort invested toward synthesizing catalysts with exclusively atomic distribution without additional nanoparticles $[8,16,21]$. Thus, the structure and activity of single atoms can be investigated without interference of nanoparticles [22-24]. Typically, catalysts with only individually dispersed metals are prepared on carbon materials with high specific surface area and heteroatom doping-especially with N $[15,17,25]$ and S [16]. Predominantly, it was shown that metal atoms bond with atoms (either $\mathrm{N}, \mathrm{S}$ or $\mathrm{C}$ ) at the edges of graphene sheets or are inserted within the vacancies in the plane of graphite sheets $[15,16,25-27]$. Heteroatom doping thus appears beneficial, but not necessary for occurrence of atomically isolated species [22,28]. Although the synthesis of a high concentration of exclusively single atoms is challenging, preparation of single atoms seems almost trivial when low concentrations of single atoms are acceptable, and clusters and nanoparticles are allowed to be present alongside single atoms. This can be achieved with different deposition techniques on various common carbon supports [22-24].

In this work, we bring into attention the coexistence of single atoms alongside nanoparticles in four commercial PGM/C catalysts $(\mathrm{PGM}=\mathrm{Ru}, \mathrm{Rh}, \mathrm{Pd}, \mathrm{Pt})$. By demonstrating the presence of single atoms in a selection of diverse and widely used commercial PGM/C catalysts, we wish to contribute towards more comprehensive understanding of the nature and behaviour of atomically dispersed metal species on carbon and their role in already established catalytic materials.

\section{Results and Discussion}

Commercial PGM/C catalysts have various different applications and, therefore, vary in their properties (e.g., carbon substrate, metal loading and average nanoparticle size) according to their intended use. For this work, four different commercial catalysts with varying metal loading were chosen, namely Ru/C, Rh/C, Pd/C (in all cases, the content was $5 \mathrm{wt} . \%$,) and Pt/C (46 wt.\%). The Pt/C catalyst is produced for electrocatalysis in a low-temperature fuel cell where typical metal loadings on carbon are between 20 and $50 \mathrm{wt} . \%$. The other three materials are commonly used in organic synthesis where loadings in the range of $0.5-10 \mathrm{wt} . \%$ are common among commercially available catalysts [2]. Producers of commercial PGM/C materials tend not to disclose structural details about the materials other than the total metal loading. In order to understand the basic morphological properties of the materials, they were analysed by scanning electron microscopy (SEM) and X-ray diffraction (XRD).

Figure 1 shows SEM images of the PGM/C materials and their X-ray diffractograms. All four diffractograms show distinct carbon peaks related to the hexagonal carbon (graphite) at $2 \theta=27^{\circ}$ (002) and $45^{\circ}$ (101) angles (PDF 04-016-6288) [29] that are widened and shifted toward lower $2 \theta$ angles due to small crystal sizes and stacking disorder [30]. However, the carbon signal in the $\mathrm{Pt} / \mathrm{C}$ sample is significantly lower. This can be explained by SEM images, which show a markedly different morphology of the carbon substrate. Pt is dispersed on a high-surface area carbon with small primary particle size (characteristic for carbon black), while all three fifth row PGM metals are deposited on a highly polydisperse carbon support (reminiscent of activated carbon) with particles ranging in size up to several micrometres $(\mathrm{Ru} / \mathrm{C}, \mathrm{Rh} / \mathrm{C})$ or tens of micrometres $(\mathrm{Pd} / \mathrm{C})$. 

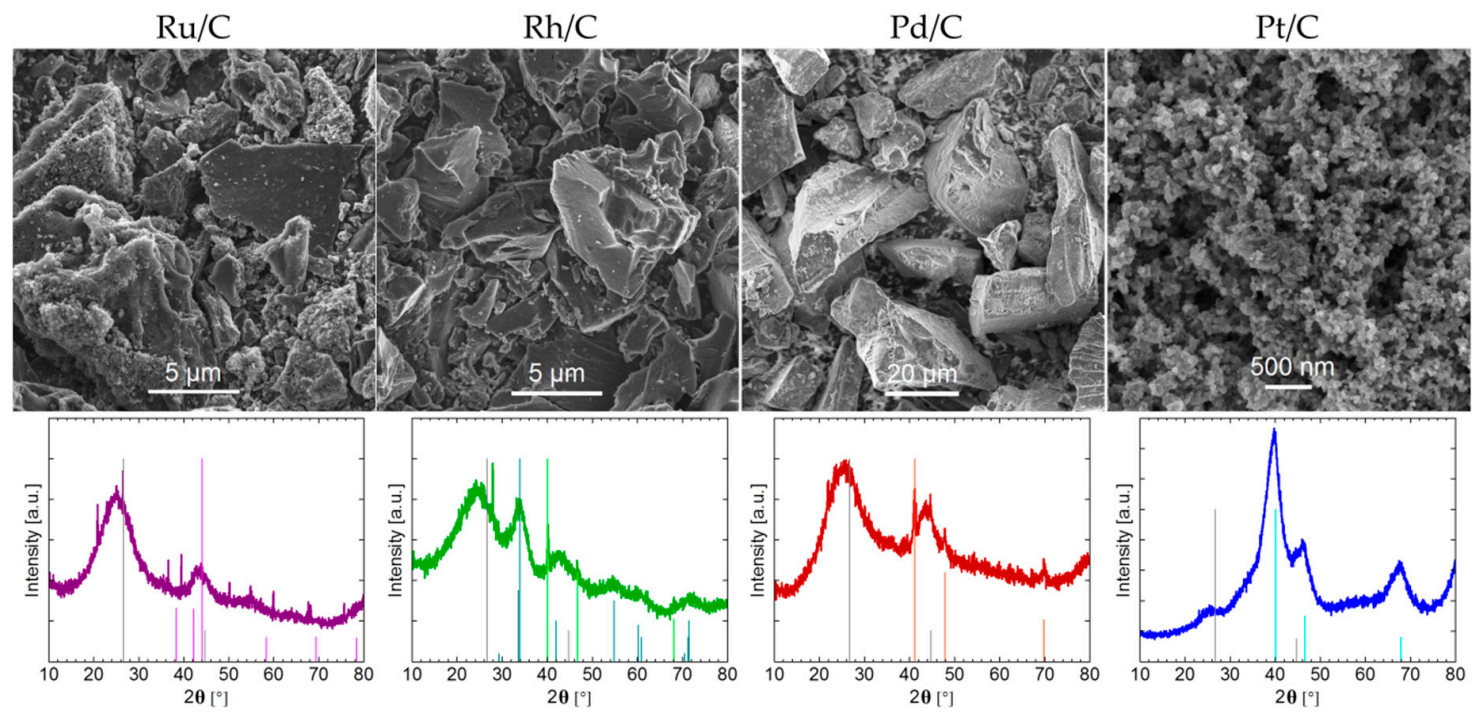

Figure 1. Characterization of commercial samples PGM/C samples: SEM images (top row) and XRD patterns (bottom row). Note that the scales in SEM micrographs vary. In addition to hexagonal graphite (PDF 04-016-6288) each XRD also displays characteristic peak positions for the corresponding metal and metal oxide: Ru—pink (PDF 00-006-0663), Rh—orange (PDF 04-016-1279), Pd—light green (PDF 04-003-0405), PdO—dark green (PDF 00-006-0515) and Pt—light blue (PDF 00-001-1194) [29].

The main role of carbon support in PGM/C catalysts is to enable a high dispersion of the metal and prevent nanoparticle aggregation. The size and distribution in the PGM/C materials was analysed by scanning transmission electron microscopy (STEM). The annular dark-field (ADF) images and bright field images in Figure 2 illustrate the typical particle distribution in the carbon support. The size distribution analysis obtained from a number of recorded images is also shown. It can be observed that both $\mathrm{Ru} / \mathrm{C}$ and $\mathrm{Rh} / \mathrm{C}$ materials consist of active metal phase that is dispersed in the form of nanoparticles with the average size of 1.3 and $1.2 \mathrm{~nm}$, respectively. Crystalline particle of this size cannot be detected in XRD patterns. Narrow peaks observed in the Rh/C diffractogram corresponding to cubic Rh suggest that there is a small number of larger nanoparticles present in the material that were not observed under STEM analysis. The low narrow peaks on the $\mathrm{Ru} / \mathrm{C}$ diffractogram that do not correspond to Ru pattern were identified as $\mathrm{SiO}_{2}$ impurities (PDF 01-070-3755) [29]. The Pd/C catalyst, on the other hand, consists of nanoparticles with an average size of $2.2 \mathrm{~nm}$. They are not evenly dispersed across the carbon support. Instead, they form large aggregates. XRD pattern reveals that, in addition to the metallic Pd phase, PdO is also present in the material. Both phases were also identified in STEM images (results not shown). Some crystalline particles were identified as Pd and others as PdO. Presumably, the catalyst is intended to be reduced in situ in the reaction mixture. $\mathrm{Pt} / \mathrm{C}$ contains nanoparticles with a similar average particle size $(2.3 \mathrm{~nm}$, close to the $2.6 \mathrm{~nm}$ value reported by the supplier) that are evenly dispersed in the high-surface area substrate. SEM, STEM and XRD results illustrate the differences between the chosen commercial materials: Different metals and their oxidation state, various types of carbon support morphologies, different particle sizes and size distributions. 


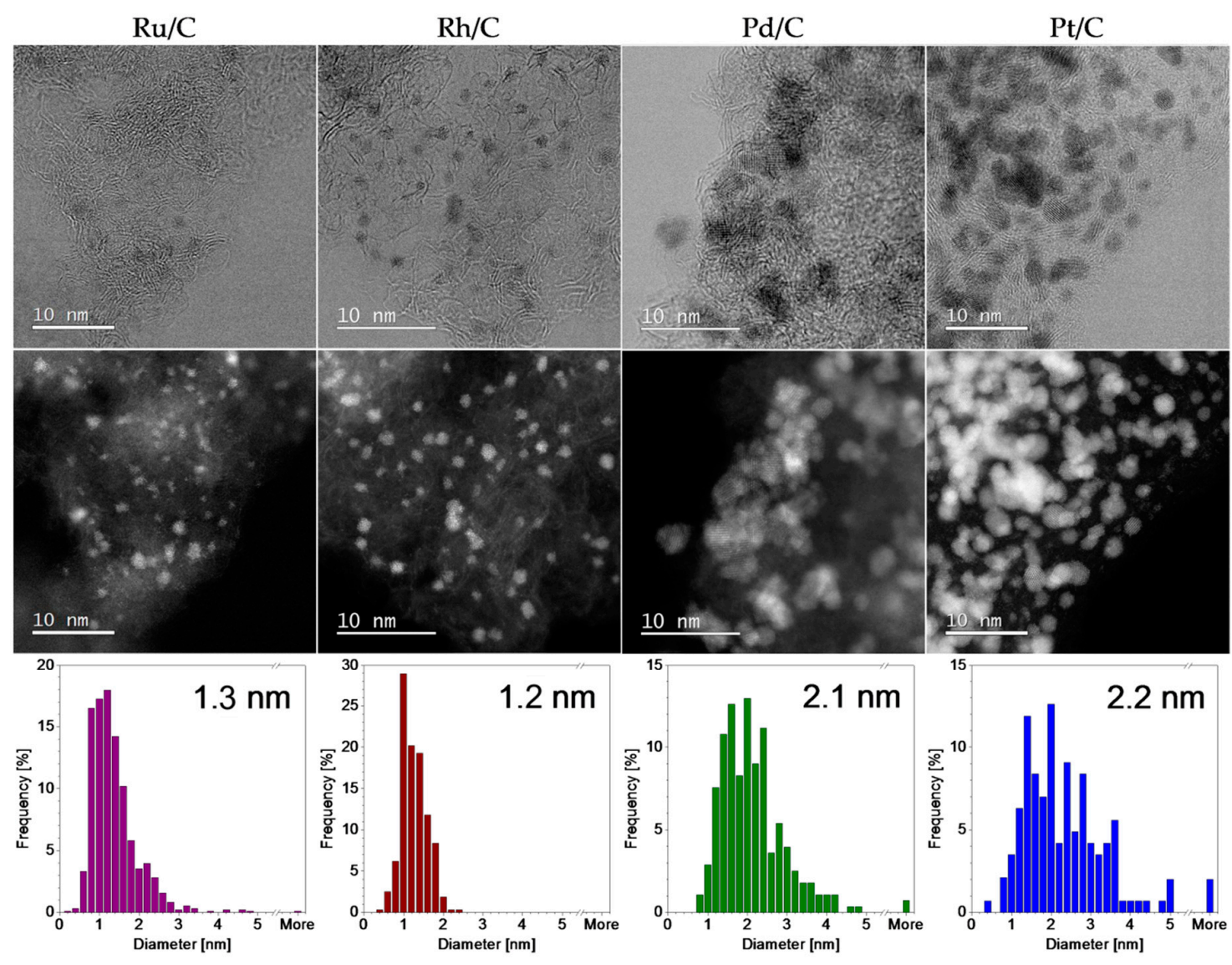

Figure 2. Representative images of the commercial PGM/C materials (PGM $=\mathrm{Ru}, \mathrm{Rh}, \mathrm{Pd}, \mathrm{Pt}$ ) acquired by STEM in bright-field (top row) and annular dark-field mode (middle row). Size distribution analyses obtained from STEM imaging (bottom row). The average sizes of the particles (diameters) are given in the size distribution diagrams.

One of the allures of single-atom catalysis as an alternative to supported nanoparticles is increasing the dispersion of the expensive metal. Dispersion is the ratio between the number of surface atoms and the total amount of metal atoms and represents the fraction of metal that is exposed to the reactants and may act catalytically. For Pt/C material, which is used as an electrocatalyst, the surface area can be determined electrochemically. Depending on the pre-treatment, the electrochemical surface area (ESA) of this commercial sample varies from approximately 75 to $100 \mathrm{~m}^{2} \mathrm{~g}^{-1} \mathrm{Pt}$ [31,32], which corresponds to approximately 30 to $40 \%$ dispersion. A commercial $\mathrm{Pt} / \mathrm{C}$ with average particle size $4.8 \mathrm{~nm}$ and ESA $56 \mathrm{~m}^{2} \mathrm{~g}^{-1} \mathrm{Pt}$ has only about $20 \%$ of its atoms at the surface, while by choosing a catalyst with smaller particles $\left(1 \mathrm{~nm}, 116 \mathrm{~m}^{2} \mathrm{~g}^{-1} \mathrm{Pt}\right.$ [32] ), the dispersion increases to about $50 \%$. This illustrates that the smaller the nanoparticles, the larger the portion of surface exposed atoms. Although it may seem a good strategy to aim for $100 \%$ dispersion when trying to reduce Pt use, increasing the dispersion by a factor of two by dispersing Pt atomically, instead of as $1 \mathrm{~nm}$-sized particles, is completely irrelevant if the atomic species cannot catalyse the reaction. Increasing the dispersion by atomic distribution should, therefore, not be the guiding principle in designing better catalysts. This, of course, does not preclude the possibility of some atomic Pt species acting as catalytically active centres for oxygen reduction reaction (ORR) or even achieving higher mass-specific activity. It merely illustrates that the logical argument that is so often used (namely, equating larger dispersion with better metal efficiency) is flawed, since it inherently makes unjustified assumptions when comparing nanoparticles and supported atoms. It presumes unmodified (or increased) activity of active centres, despite the change of the active centre structure and unmodified (or increased) number of active centres per number of surface-exposed atoms. 
The images in Figure 2 only show metallic nanoparticles, while higher magnification images in Figure 3 reveal that all four investigated commercial catalysts contain significant numbers of atomically dispersed metal species. Several different areas of each material were investigated, and single atoms were consistently found in the materials. For single-atom imaging, the ADF detector is preferred over the bright field detector because it can accept large-angle scattered electrons [33-35], especially in carbon substrate, because of the large $Z$-contrast between carbon $(Z=6)$ and PGM atoms $(Z=44,45$, 46 and 78 for $\mathrm{Ru}, \mathrm{Rh}, \mathrm{Pd}$ and $\mathrm{Pt}$, respectively). However, the observed contrast strongly depends on substrate thickness. To visualize atomic species, areas of the carbon substrates were chosen at the edges of carbon particles or at thin areas of the carbon particles.
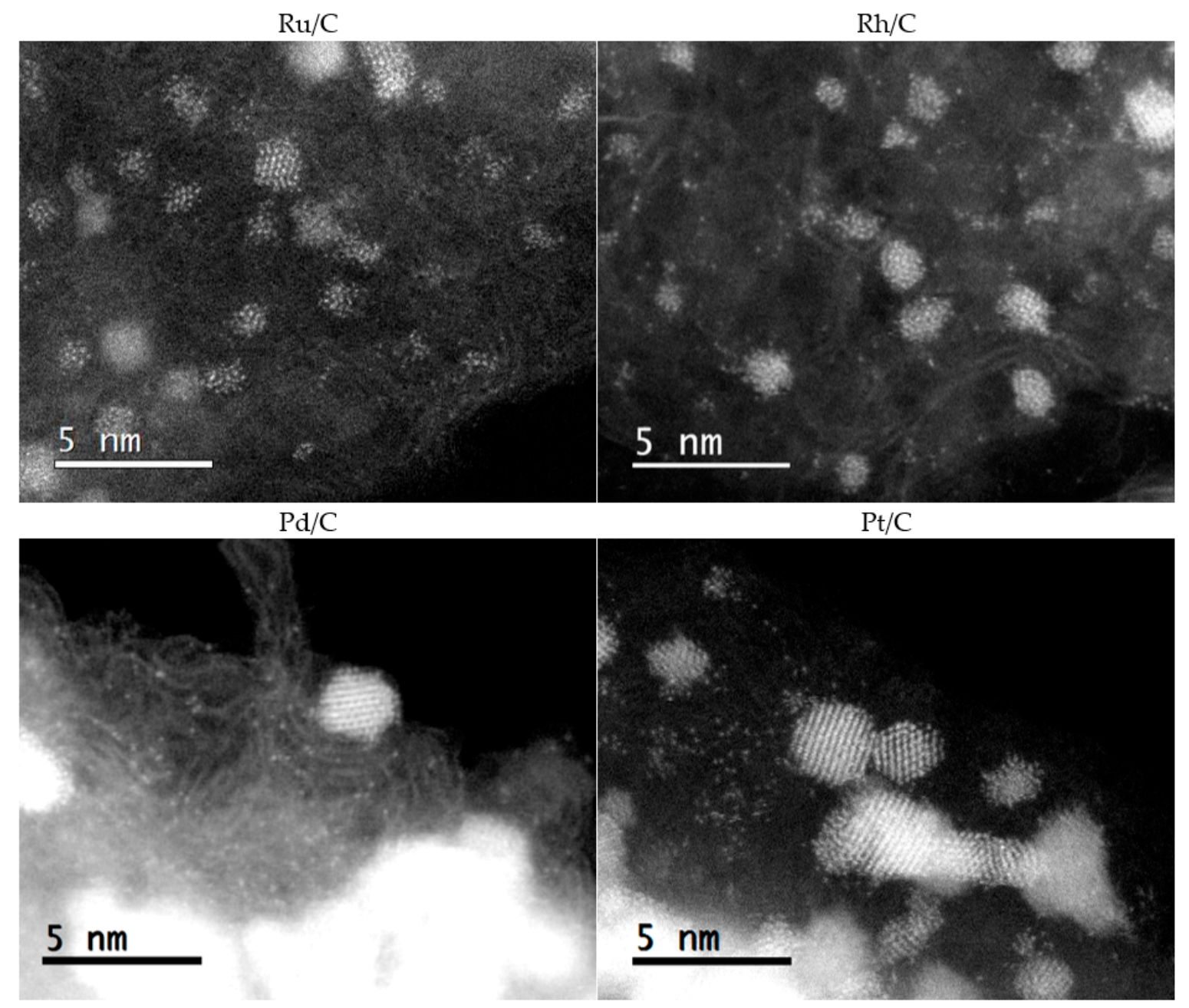

Figure 3. AC-STEM images of $\mathrm{PGM} / \mathrm{C}$ catalysts $(\mathrm{PGM}=\mathrm{Ru}, \mathrm{Rh}, \mathrm{Pd}, \mathrm{Pt})$ recorded in annular dark-field mode.

It is well-known that irradiation by the electron beam in STEM can cause structural damage to clusters and nanoparticles due to electron dose accumulation [36]. This may result in sputtering of single atoms or even a complete breakdown of smaller clusters into atomic species [36]. Several precautions were taken during imaging in order to avoid unintentional sputtering. A relatively low-voltage of $80 \mathrm{kV}$ and a beam current of $\sim 14.5 \mathrm{pA}$ was used. Additionally, settings and focus were adjusted outside of the area intended for investigation before the image was recorded on the area that had previously not been exposed to the beam. Single atoms were observed, even at relatively low magnification (around $5 \times 10^{6}$ ), where electron dosage per unit area was very low. Moreover, single atomic species were observed not only in the vicinity of nanoparticles (where they would be expected if they were caused by sputtering), but also in areas of the carbon material that were 
several nanometres away from the nearest metal clusters. All this confirms that the majority of the observed atoms were indeed characteristic for the material and were not sputtered from nanoparticles by electron beam irradiation.

These STEM observations clearly reveal that single atoms are present alongside nanoparticles in commercial PGM/C catalysts with different types of metals ( $\mathrm{Ru}, \mathrm{Rh}, \mathrm{Pd}, \mathrm{Pt}$ ), loadings (5 wt.\% or $46 \mathrm{wt} . \%$ ), particle sizes (1.3 to $2.2 \mathrm{~nm}$ ) and carbon supports (high-surface area carbon and large polydisperse carbon particles). If the investigated four materials can be thought of as typical and representative commercial catalysts, it can be presumed that single atoms may be ubiquitously present alongside nanoparticles in many other commercial and laboratory-synthesized carbon-supported metal catalysts. Surprisingly, such findings are not commonly reported. Even when similar materials are investigated with sub-ångström resolution STEM, individual atoms may be overlooked if they are not investigated at high enough magnifications or under optimal conditions (annular dark field mode instead of bright field). Large particles of carbon support may also cause a high background signal upon which individual atoms may not be notable. Most importantly, even when individual supported atoms are detected, they may be considered to be caused by beam irradiation damage or just simply not relevant for the conducted research and, therefore, not shown or remarked on in the publications [37].

In addition to the commercial materials discussed in this work, our experience with STEM analysis of carbon-supported catalysts suggested that atomic species are indeed a common companion to metal nanoparticles. For example, we detected single atoms in a commercial PtRu/C catalyst [38] and commercial $\mathrm{Pt}-\mathrm{SnO}_{2} / \mathrm{C}$ catalyst [39] on a high-surface area nitrogen-doped carbon alongside $\mathrm{Pt}$ nanoparticles [32] and on a graphite rod after electrochemical deposition of $\mathrm{Ag}$ [40].

Simple wet chemical deposition techniques that are proven successful for preparing single atoms [14] are also widely used for depositing nanoparticle-based catalysts. Therefore, it stands to reason that substantial concentrations of single atoms may be present in many carbon-supported materials and not only in cases when high-surface area carbons or heteroatom-doped carbons are used as substrates. Here, we suggest some possible origins of single-atom formation during the synthesis of the catalysts. It should be noted that details of preparation are understandably not provided by the producers and, therefore, the following discussion can only be general and not specific with respect to the characterized materials. In a typical deposition process, the metal precursor is deposited on the carbon substrate either by adsorption or impregnation. In addition to ionic attraction to charged surface groups, the metal precursor may also form specific chemical interactions with sites in the carbon support such as edges or vacancies. The likelihood of chemical bonding increases when heteroatoms are present. Chemisorption thus results in atomic distribution of the precursor, though impregnation with high precursor loadings probably also leaves behind precursor particles after the solvent has dried. Commonly, the synthesis also includes a reduction step (although the metal can also be provided in a partially oxidized state, as in the case of the investigated $\mathrm{Pd} / \mathrm{C}$ catalyst). Reduction can be carried out by reducing agents in the solution or a high-temperature annealing step in either inert or reductive atmosphere. It is unclear how supported atomic species may differ from the precursor and how they may be affected by chemical reduction agents. Thermal treatment offers more clear ideas on possible preservation or formation of atomic species. Higher temperatures increase the mobility of metal species on the substrate surface and thus enable aggregation. When the mobile species move across the support surface, they are, in fact, single atoms. It can be imagined that, at any moment during annealing, there is a certain surface concentration of single atoms alongside nanoparticles that may be preserved after cooling the sample to room temperature. Concentration and type of anchoring sites for single atoms on the carbon surface may have an important role during catalyst synthesis (nucleation, sintering during annealing).

The visual confirmation of significant amounts of single atoms alongside nanoparticles in commercial PGM/C catalysts poses a number of questions regarding their nature and behaviour. An important piece of information would be a quantitative estimation of the number of single atoms 
with respect to the nanoparticles. For model catalysts with flat surfaces, this estimation can be made on the basis of STEM images [41], but for materials with large three-dimensional carbon particles, such quantifications would be extremely unreliable. Another dilemma would focus on the role these atomic species play in the overall catalytic behaviour of the material. Based on the number of reportedly active single atoms, it is conceivable that, in some cases, the atomic species may be catalytically active in addition to, or instead of, the active sites on the nanoparticles. However, as discussed above, due to fundamentally different natures of atomic and metallic species, in most cases atomic species cannot be expected to display similar activity for a given reaction as clusters or nanoparticles. They may, however, strongly influence the properties of the material in other ways. For example, one of the mechanisms of supported catalyst sintering (loss of surface area) is Ostwald ripening, which occurs through mobility of atomic species from smaller to larger metal particles and is driven by higher thermodynamic stability of larger nanoparticles [42]. This process can occur not only during thermal steps of the catalyst synthesis as mentioned earlier, but more importantly, under reaction conditions during the catalytic process [41]. Mass transport can occur either through liquid or gas phase of the reaction mixture or across the surface of the substrate, i.e., as single atoms [43]. Another important issue is the stability of isolated metal species under reaction conditions. Here, we refer not only to stability against aggregation, but also to stability against detachment (leaching) and carbon corrosion. On the other hand, the anchoring sites on carbon support can re-adsorb metal atoms leached from the nanoparticles [32].

Dilemmas and questions such as those raised above about the role of single atoms in traditional nanoparticle-based materials have not yet been introduced into the growing field of single-atom catalysis. It would, however, be highly beneficial to increase our understanding of their nature and behaviour under catalytic conditions. It may be imagined that atomic species are ubiquitously present in other commercial $\mathrm{M} / \mathrm{C}$ catalysts and laboratory-prepared materials that follow similar preparation methods (e.g., wet deposition followed by annealing). The concentration of single atoms most likely varies significantly with different supports, metals and preparation techniques, but the largest amounts may be expected for metals with small particle size, dispersed on high-surface area carbons with heteroatom doping [16,32]. Some reports within the single-atom community that show nanoparticles and single atoms in the same material relate the atomic distribution to positive effects on observed catalytic activity $[15,22,23]$. This is certainly possible; any observed activity, of course, depends on the nature of the metal, support, the studied reaction, etc. However, we would like to suggest that single atoms may accompany many supported metal nanoparticles. Their unexpected discovery may lead some researchers to overestimate the uniqueness of atomic distribution in the studied materials. On the other hand, researchers unaware of their possible existence may be able to gain better understanding of their catalysts provided they pay attention not only to nanoparticles, but also to atomic metal species.

The first step toward addressing all the raised questions is to understand the nature of the atomic species, i.e., their electronic structure and type of bonding with the support. Figure 4 shows single atoms in a $\mathrm{Pd} / \mathrm{C}$ material at high magnification on a very thin edge of carbon particles that only consist of several entangled graphene sheets. The image suggests possible anchoring sites for metal atoms in the carbon material. It can be observed that some Pd atoms tend to appear alongside bright lines that represent edges of graphene sheets (oval shapes in Figure 4), while it is also possible to find atoms that appear to be imbedded in the plane of graphene sheet (square in Figure 4). This is in accordance with literature that reports anchoring sites for metal atoms at defect sites (such as vacancies) and alongside graphene edges [24,44]. 
(a)

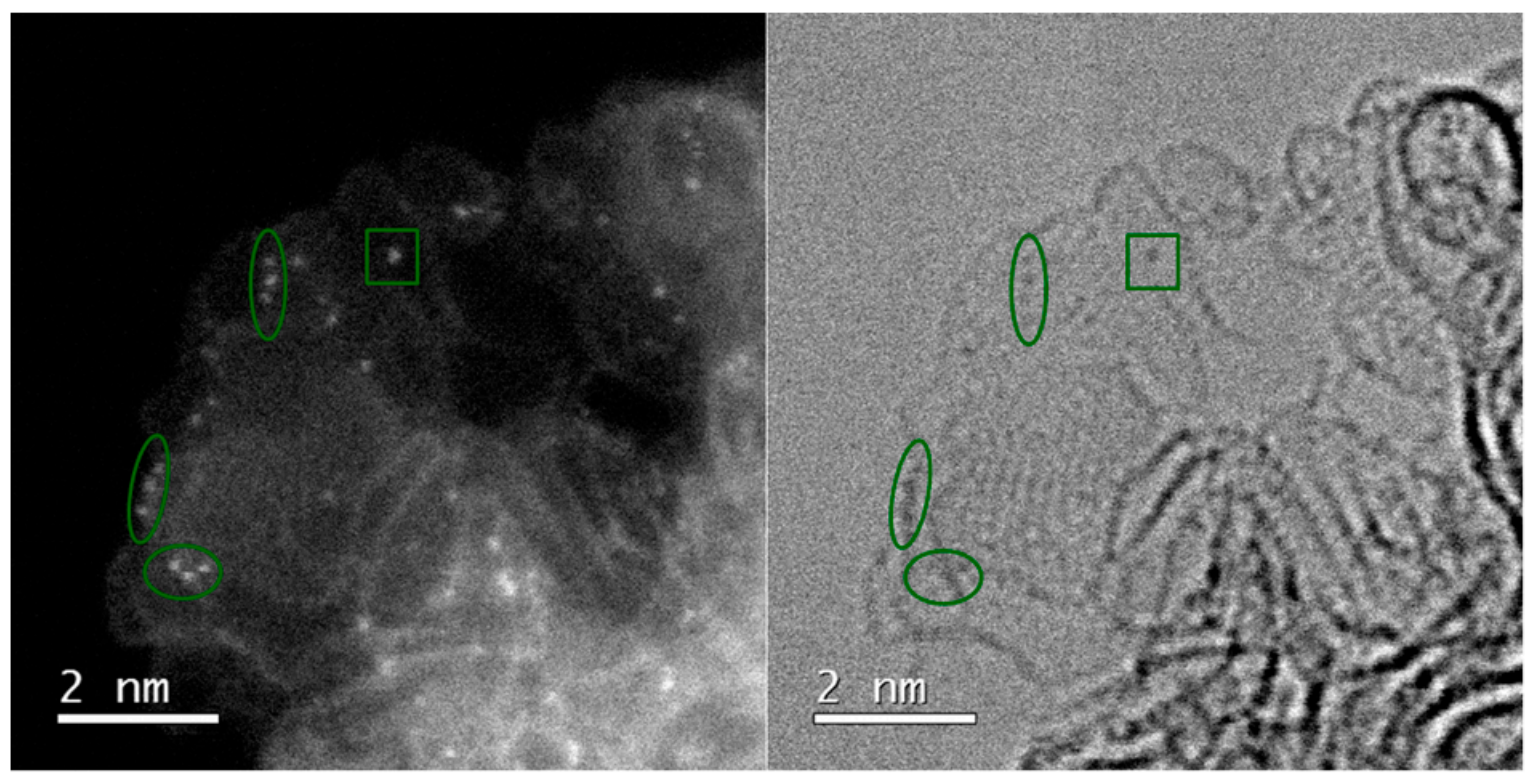

Figure 4. Single atoms of Pd in Pd/C catalyst in (a) annular dark-field and (b) bright-field mode.

The rich chemistry of carbon supports combined with coordination chemistry of metals thus offers great variability in the nature of atomic species and poses great challenges and opportunities for the future work in single-atom catalysis.

\section{Materials and Methods}

Four commercially available PGM/C materials were investigated, namely Ru/C (5 wt.\% Ru, Sigma-Aldrich, St. Louis, MO, USA; 206180, powder), Rh/C (5 wt\% Rh, Riogen, Mullica Hill, NJ, USA; 0127-CRhA05, pellets), Pd/C (Sigma-Aldrich, St. Louis, MO, USA; 5 wt.\%, 520837, powder) and $\mathrm{Pt} / \mathrm{C}$ (Tanaka Kikinzoku Kogyo, Tokyo, Japan; $46 \mathrm{wt.} \%$, TEC10E50E, powder). The Rh/C material was supplied in the form of pellets and was milled and sieved before further characterization. The sub-100 $\mu \mathrm{m}$ fraction was investigated. The other catalysts were supplied in powder form.

Their morphology was investigated under Zeiss field emission scanning electron microscope FE-SEM SUPRA 35 VP (Carl Zeiss, Oberkochen, Germany). X-ray diffractograms of the catalysts were recorded on flat disc-like Si sample holder with an X-ray powder diffractometer PANalytical X'Pert PRO MPD (PANalytical B.V., Almelo, The Netherlands) with radiation wavelength CuK $\alpha 1=1.5406 \AA$ in alpha1 configuration with Johansson monochromator on the primary side. The diffractograms were recorded with $0.034^{\circ}$ resolution and $100 \mathrm{~s}$ signal integration time in the $2 \theta$ range from $10^{\circ}$ to $80^{\circ}$ with a scanning $X^{\prime}$ Celerator detector $\left(2.122^{\circ} 2 \theta\right)$. The 0.02 rad Soller slits and $10 \mathrm{~mm}$ divergent slits were used together with a $10 \mathrm{~mm}$ beam mask. Phase identification was performed in the X'Pert HighScore Plus program using the International Centre for Diffraction Data (ICDD) PDF-4+ 2016 database [29].

The materials were also characterized with an atomic resolution aberration corrected scanning transmission electron microscope ARM 200CF (JEOL, Tokyo, Japan) working at $80 \mathrm{kV}$ accelerating voltage and $\sim 14.5 \mathrm{pA}$ beam current. ADF images were taken with $45-180 \mathrm{mrad}$ and with $68-280 \mathrm{mrad}$ for inner-outer semi-angles. The convergence semi-angle was set to $25 \mathrm{mrad}$. Sample preparation was carried out by diluting the powder in ethanol and drop-casting the solution with a pipette on lacey carbon coated $\mathrm{Cu}$ grids.

\section{Conclusions}

Single atoms were observed in some commonly used commercial nanoparticle-based catalysts with PGM metals on carbon support. Although sub-ångström resolution AC-STEM is becoming 
progressively more accessible, the ubiquitous presence of single atoms alongside nanoparticles on carbon supports is not yet widely recognized, let alone taken into account in catalyst evaluation. We show that atomically dispersed metals accompany nanoparticles of different metals $(\mathrm{Ru}, \mathrm{Rh}, \mathrm{Pd}$ and $\mathrm{Pt})$ with different metal loadings ( $5 \mathrm{wt} . \%$ and $46 \mathrm{wt} . \%$ ), different particle size distribution and on various carbon supports. We believe that the present results strongly suggest that any thorough study of catalytic activity of given materials needs to address the presence of single atoms. In that respect, the new understanding about atomically dispersed metals can contribute greatly not only to developing new types of active catalysts with low metal loadings, but also to the understanding of the traditional nanoparticle-based supported catalyst. Without that the possible (significant) contribution of the so-far "invisible" single atoms and atomic clusters will remain overlooked.

Author Contributions: U.P. conceptualized the idea for the manuscript and prepared the original draft. F.R.-Z. recorded AC-STEM images, performed size-distribution analysis and reviewed the manuscript. M.B. supervised the project and reviewed the manuscript. M.G. supervised the project and reviewed the manuscript.

Funding: This research was funded by the Slovenian Research Agency through research programme P2-0393 and the young researcher programme (U. Petek). Part of the work was carried out within the NATO SfP Project EAP.SFPP 984925-“DURAPEM".

Acknowledgments: XRDs were recorded by E. Kranjc (Department of Inorganic Chemistry and Technology, National Institute of Chemistry, Slovenia). The authors also thank A. Bjelić and M. Grilc (Laboratory of Catalysis and Chemical Reaction Engineering, National Institute of Chemistry, Slovenia) for providing some of the analysed materials.

Conflicts of Interest: The authors declare no conflict of interest.

\section{References}

1. Auer, E.; Freund, A.; Pietsch, J.; Tacke, T. Carbons as supports for industrial precious metal catalysts. Appl. Catal. A Gen. 1998, 173, 259-271. [CrossRef]

2. Blaser, H.-U.; Indolese, A.; Schnyder, A.; Steiner, H.; Studer, M. Supported palladium catalysts for fine chemicals synthesis. J. Mol. Catal. A Chem. 2001, 173, 3-18. [CrossRef]

3. Shao, M. Electrocatalysis in fuel cells. Catalysts 2015, 5, 2115-2121. [CrossRef]

4. Bezerra, C.W.B.; Zhang, L.; Lee, K.; Liu, H.; Marques, A.L.B.; Marques, E.P.; Wang, H.; Zhang, J. A review of Fe-N/C and Co-N/C catalysts for the oxygen reduction reaction. Electrochim. Acta 2008, 53, 4937-4951. [CrossRef]

5. Li, J.; Jaouen, F. Structure and activity of metal-centered coordination sites in pyrolyzed metal-nitrogen-carbon catalysts for the electrochemical reduction of $\mathrm{O}_{2}$. Curr. Opin. Electrochem. 2018, 9, 198-206. [CrossRef]

6. Liang, S.; Hao, C.; Shi, Y. The power of single-atom catalysis. ChemCatChem 2015, 7, 2559-2567. [CrossRef]

7. Yang, X.-F.; Wang, A.; Qiao, B.; Li, J.; Liu, J.; Zhang, T. Single-atom catalysts: A new frontier in heterogeneous catalysis. Acc. Chem. Res. 2013, 46, 1740-1748. [CrossRef]

8. Liu, J. Catalysis by supported single metal atoms. ACS Catal. 2017, 7, 34-59. [CrossRef]

9. Haider, M.; Uhlemann, S.; Schwan, E.; Rose, H.; Kabius, B.; Urban, K. Electron microscopy image enhanced. Nature 1998, 392, 768-769. [CrossRef]

10. Batson, P.E.; Dellby, N.; Krivanek, O.L. Sub-ångstrom resolution using aberration corrected electron optics. Nature 2002, 418, 617-620. [CrossRef]

11. Li, H.; Zhang, H.-X.; Yan, X.-L.; Xu, B.-S.; Guo, J.-J. Carbon-supported metal single atom catalysts. New Carbon Mater. 2018, 33, 1-11. [CrossRef]

12. Mitchell, S.; Vorobyeva, E.; Pérez-Ramírez, J. The multifaceted reactivity of single-atom heterogeneous catalysts. Angew. Chem. Int. Ed. 2018, 57, 15316-15329. [CrossRef] [PubMed]

13. Yan, H.; Su, C.; He, J.; Chen, W. Single-atom catalysts and their applications in organic chemistry. J. Mater. Chem. A 2018, 6, 8793-8814. [CrossRef]

14. Su, J.; Ge, R.; Dong, Y.; Hao, F.; Chen, L. Recent progress in single-atom electrocatalysts: Concept, synthesis, and applications in clean energy conversion. J. Mater. Chem. A 2018, 6, 14025-14042. [CrossRef] 
15. Bulushev, D.A.; Zacharska, M.; Lisitsyn, A.S.; Podyacheva, O.Y.; Hage, F.S.; Ramasse, Q.M.; Bangert, U.; Bulusheva, L.G. Single atoms of Pt-group metals stabilized by $\mathrm{N}$-doped carbon nanofibers for efficient hydrogen production from formic acid. ACS Catal. 2016, 6, 3442-3451. [CrossRef]

16. Choi, C.H.; Kim, M.; Kwon, H.C.; Cho, S.J.; Yun, S.; Kim, H.-T.; Mayrhofer, K.J.J.; Kim, H.; Choi, M. Tuning selectivity of electrochemical reactions by atomically dispersed platinum catalyst. Nat. Commun. 2016, 7, 10922. [CrossRef] [PubMed]

17. Podyacheva, O.Y.; Bulushev, D.A.; Suboch, A.N.; Svintsitskiy, D.A.; Lisitsyn, A.S.; Modin, E.; Chuvilin, A.; Gerasimov, E.Y.; Sobolev, V.I.; Parmon, V.N. Highly stable single-atom catalyst with ionic Pd active sites supported on N-doped carbon nanotubes for formic acid decomposition. ChemSusChem 2018, 11, 3724-3727. [CrossRef] [PubMed]

18. Molleman, B.; Hiemstra, T. Size and shape dependency of the surface energy of metallic nanoparticles: Unifying the atomic and thermodynamic approaches. Phys. Chem. Chem. Phys. 2018, 20, 20575-20587. [CrossRef] [PubMed]

19. Vollath, D.; Fischer, F.D.; Holec, D. Surface energy of nanoparticles_-Influence of particle size and structure. Beilstein J. Nanotechnol. 2018, 9, 2265-2276. [CrossRef] [PubMed]

20. Chen, Y.; Huang, Z.; Ma, Z.; Chen, J.; Tang, X. Fabrication, characterization, and stability of supported single-atom catalysts. Catal. Sci. Technol. 2017, 7, 4250-4258. [CrossRef]

21. Li, T.; Liu, J.; Song, Y.; Wang, F. Photochemical solid-phase synthesis of platinum single atoms on nitrogen-doped carbon with high loading as bifunctional catalysts for hydrogen evolution and oxygen reduction reactions. ACS Catal. 2018, 8, 8450-8458. [CrossRef]

22. Inoue, H.; Ishii, T.; Kannari, N.; Ozaki, J.I. Electrochemical properties of an atomically dispersed platinum catalyst formed on a heat-treated carbon support. ChemistrySelect 2016, 1, 3189-3196. [CrossRef]

23. Cheng, N.; Stambula, S.; Wang, D.; Banis, M.N.; Liu, J.; Riese, A.; Xiao, B.; Li, R.; Sham, T.-K.; Liu, L.-M.; et al. Platinum single-atom and cluster catalysis of the hydrogen evolution reaction. Nat. Commun. 2016, 7, 13638. [CrossRef] [PubMed]

24. Sun, S.; Zhang, G.; Gauquelin, N.; Chen, N.; Zhou, J.; Yang, S.; Chen, W.; Meng, X.; Geng, D.; Banis, M.N.; et al. Single-atom catalysis using Pt/graphene achieved through atomic layer deposition. Sci. Rep. 2013, 3, 1775. [CrossRef]

25. Zitolo, A.; Goellner, V.; Armel, V.; Sougrati, M.-T.; Mineva, T.; Stievano, L.; Fonda, E.; Jaouen, F. Identification of catalytic sites for oxygen reduction in iron- and nitrogen-doped graphene materials. Nat. Mater. 2015, 14, 937-942. [CrossRef]

26. Fei, H.; Dong, J.; Feng, Y.; Allen, C.S.; Wan, C.; Volosskiy, B.; Li, M.; Zhao, Z.; Wang, Y.; Sun, H.; et al. General synthesis and definitive structural identification of MN4C4 single-atom catalysts with tunable electrocatalytic activities. Nat. Catal. 2018, 1, 63-72. [CrossRef]

27. Liu, J.; Jiao, M.; Mei, B.; Tong, Y.; Li, Y.; Ruan, M.; Song, P.; Sun, G.; Jiang, L.; Wang, Y.; et al. Carbon-supported divacancy-anchored platinum single-atom electrocatalysts with superhigh Pt utilization for the oxygen reduction reaction. Angew. Chem. Int. Ed. 2019, 58, 1163-1167. [CrossRef] [PubMed]

28. Krasheninnikov, A.V.; Lehtinen, P.O.; Foster, A.S.; Pyykkö, P.; Nieminen, R.M. Embedding transition-metal atoms in graphene: Structure, bonding, and magnetism. Phys. Rev. Lett. 2009, 102, 126807. [CrossRef] [PubMed]

29. ICDD. PDF-4+ 2016 (Database); Kabekkodu, S., Ed.; International Centre for Diffraction Data: Newtown Square, PA, USA, 2016.

30. Li, Z.Q.; Lu, C.J.; Xia, Z.P.; Zhou, Y.; Luo, Z. X-ray diffraction patterns of graphite and turbostratic carbon. Carbon 2007, 45, 1686-1695. [CrossRef]

31. Gatalo, M.; Jovanovič, P.; Polymeros, G.; Grote, J.-P.; Pavlišič, A.; Ruiz-Zepeda, F.; Šelih, V.S.; Šala, M.; Hočevar, S.; Bele, M.; et al. Positive effect of surface doping with Au on the stability of Pt-based electrocatalysts. ACS Catal. 2016, 6, 1630-1634. [CrossRef]

32. Jovanovič, P.; Petek, U.; Hodnik, N.; Ruiz-Zepeda, F.; Gatalo, M.; Šala, M.; Šelih, V.S.; Fellinger, T.P.; Gaberšček, M. Importance of non-intrinsic platinum dissolution in Pt/C composite fuel cell catalysts. Phys. Chem. Chem. Phys. 2017, 19, 21446-21452. [CrossRef] [PubMed]

33. Crewe, A.V.; Wall, J.; Langmore, J. Visibility of single atoms. Science 1970, 168, 1338-1340. [CrossRef] [PubMed] 
34. Treacy, M.M.J. Z dependence of electron scattering by single atoms into annular dark-field detectors. Microsc. Microanal. 2011, 17, 847-858. [CrossRef] [PubMed]

35. Pennycook, S.J.; Chisholm, M.F.; Lupini, A.R.; Varela, M.; Borisevich, A.Y.; Oxley, M.P.; Luo, W.D.; van Benthem, K.; Oh, S.H.; Sales, D.L.; et al. Aberration-corrected scanning transmission electron microscopy: From atomic imaging and analysis to solving energy problems. Philos. Trans. R. Soc. A Math. Phys. Eng. Sci. 2009, 367, 3709-3733. [CrossRef] [PubMed]

36. Egerton, R.F.; Li, P.; Malac, M. Radiation damage in the TEM and SEM. Micron 2004, 35, 399-409. [CrossRef] [PubMed]

37. Asoro, M.A.; Kovar, D.; Shao-Horn, Y.; Allard, L.F.; Ferreira, P.J. Coalescence and sintering of Pt nanoparticles: In situ observation by aberration-corrected HAADF STEM. Nanotechnology 2010, 21, 025701. [CrossRef] [PubMed]

38. Jovanovič, P.; Šelih, V.S.; Šala, M.; Hočevar, S.; Ruiz-Zepeda, F.; Hodnik, N.; Bele, M.; Gaberšček, M. Potentiodynamic dissolution study of PtRu/C electrocatalyst in the presence of methanol. Electrochim. Acta 2016, 211, 851-859. [CrossRef]

39. Jovanovič, P.; Ruiz-Zepeda, F.; Šala, M.; Hodnik, N. Atomic scale insights into electrochemical dissolution of Janus $\mathrm{Pt}-\mathrm{SnO}_{2}$ nanoparticles in the presence of ethanol in acidic media: An IL-STEM and EFC-ICP-MS study. J. Phys. Chem. C 2018, 122, 10050-10058. [CrossRef]

40. Vanrenterghem, B.; Bele, M.; Zepeda, F.R.; Šala, M.; Hodnik, N.; Breugelmans, T. Cutting the Gordian Knot of electrodeposition via controlled cathodic corrosion enabling the production of supported metal nanoparticles below $5 \mathrm{~nm}$. Appl. Catal. B Environ. 2018, 226, 396-402. [CrossRef]

41. Boyes, E.D.; Gai, P.L. Visualising reacting single atoms under controlled conditions: Advances in atomic resolution in situ Environmental (Scanning) Transmission Electron Microscopy (E(S)TEM). C. R. Phys. 2014, 15, 200-213. [CrossRef]

42. Hansen, T.W.; DeLaRiva, A.T.; Challa, S.R.; Datye, A.K. Sintering of catalytic nanoparticles: Particle migration or Ostwald ripening? Acc. Chem. Res. 2013, 46, 1720-1730. [CrossRef] [PubMed]

43. José-Yacamán, M.; Gutierrez-Wing, C.; Miki, M.; Yang, D.Q.; Piyakis, K.N.; Sacher, E. Surface diffusion and coalescence of mobile metal nanoparticles. J. Phys. Chem. B 2005, 109, 9703-9711. [CrossRef] [PubMed]

44. Wang, H.; Wang, Q.; Cheng, Y.; Li, K.; Yao, Y.; Zhang, Q.; Dong, C.; Wang, P.; Schwingenschlögl, U.; Yang, W.; et al. Doping monolayer graphene with single atom substitutions. Nano Lett. 2012, 12, 141-144. [CrossRef] [PubMed] 